Artigo

\title{
Uma exploração do Hexágono de Dürer com professores de Matemática da Educação Básica
}

\author{
An exploration of the Dürer Hexagon with Mathematics teachers of Basic Education \\ Una exploración del Hexágono de Dürer con profesores de Matemática de Educación \\ Básica
}

\author{
Fabrícia de Carvalho Paixão ${ }^{1}$ \\ (D) [0000-0002-9611-1156] \\ Mariana Moran² \\ [0000-0001-8887-8560] \\ Veridiana Rezende ${ }^{3}$ \\ [0000-0002-4158-2196]
}

\begin{abstract}
Resumo
Neste trabalho apresentamos uma análise de tarefas matemáticas elaboradas por professores de Matemática da Educação Básica, baseadas no Hexágono de Dürer. Com esta pesquisa tivemos a intenção de responder as seguintes questões: os professores, colaboradores desta pesquisa, exploram diferentes Registros de Representação Semiótica ao elaborarem atividades relacionadas à Geometria Fractal? Se sim, o processo de conversão entre estas representações ocorre? De que maneira? Durante a análise da pesquisa nos baseamos, principalmente, na teoria dos Registros de Representação Semiótica de Raymond Duval, com o olhar para a Geometria Fractal. Os sujeitos da pesquisa foram 14 professores de Matemática da Educação Básica, participantes do Programa de Desenvolvimento Educacional (PDE) do Estado do Paraná. Para o desenvolvimento da pesquisa, primeiramente realizamos com estes sujeitos a construção do Hexágono de Dürer com o auxílio do software GeoGebra, e propomos um questionário que foi respondido pelos professores. Tivemos como principal resultado que a representação simbólica numérica foi a mais indicada pelos professores, e, deste modo, a conversão do registro figural para o simbólico numérico teve destaque, e a representação simbólico algébrica foi a menos sugerida pelos professores.
\end{abstract}

Palavras-chave: Ensino de Matemática. Representação Semiótica. Hexágono de Dürer. Software GeoGebra.

\section{Abstract}

In this work we present an analysis of mathematical tasks elaborated by Mathematics teachers of Basic Education, based on the Dürer Hexagon. With this research we intend to answer the following questions: Do the teachers, collaborators of this research, explore different Registers of Semiotic Representation when elaborating activities related to Fractal Geometry? If so, does the process of

\footnotetext{
${ }^{1}$ fah-carvalho@hotmail.com, especialista em Educação Especial Inclusiva, Cianorte/Paraná/Brasil.

${ }^{2}$ marianamoranbar@gmail.com, doutora, docente do Departamento de Matemática da Universidade Estadual de Maringá (UEM) e do Programa de Pós-graduação em Educação Matemática (PRPGEM) da Universidade Estadual do Paraná (UNESPAR), Universidade Estadual de Maringá - UEM, Maringá/Paraná/Brasil.

3 rezendeveridiana@gmail.com, doutora, docente do Colegiado de Matemática e do Programa de Pósgraduação em Educação Matemática (PRPGEM), Universidade Estadual do Paraná - Unespar, Campo Mourão/Paraná/ Brasil.
} 
conversion between these representations occur? In what way? During the analysis of the research we rely mainly on Raymond Duval's Theory of Semiotic Representation Records, with a look at Fractal Geometry. The subjects of the research were 14 teachers of Mathematics of Basic Education, participants of the Program of Educational Development (PDE) of the State of Paraná. For the development of the research, we first performed with these subjects the construction of the Dürer Hexagon with the help of GeoGebra software, and we proposed a questionnaire that was answered by the teachers. We had as main result that the numerical symbolic representation was the most indicated by the teachers, and, thus, the conversion of the figural register to the numerical symbolic was highlighted, and the algebraic symbolic representation was the least suggested by the teachers.

Keywords: Mathematics Teaching. Semiotic Representation. Hexagon Dürer. GeoGebra Software.

\section{Resumen}

En este artículo presentamos un análisis de tareas matemáticas desarrollado por profesores de Matemáticas en Educación Básica, basado en el Hexágono de Dürer. Con esta investigación tuvimos la intención de responder las siguientes preguntas: ¿̇los maestros, colaboradores en esta investigación, exploran diferentes registros de representación semiótica al desarrollar actividades relacionadas con la geometría fractal? Si es así, ¿̇ocurre el proceso de conversión entre estas representaciones? ¿De que manera? Durante el análisis de la investigación, nos basamos principalmente en la teoría de Raymond Duval de los registros de representación semiótica, con miras a la geometría fractal. Los sujetos de investigación fueron 14 profesores de Matemáticas de Educación Básica, que participaron en el Programa de Desarrollo Educativo (PDE) del Estado de Paraná. Para el desarrollo de la investigación, primero realizamos con estos temas la construcción del Hexágono de Durero con la ayuda del software GeoGebra, y propusimos un cuestionario que fue respondido por los maestros. Tuvimos como resultado principal que la representación simbólica numérica fue la más indicada por los maestros y, por lo tanto, la conversión del registro figurativo a la simbólica numérica fue resaltada, y la representación simbólica algebraica fue la menos sugerida por los maestros.

Palabras claves: Enseñanza de las matemáticas. Representación semiótica. Hexágono de Dürer. Software GeoGebra.

\section{Introdução}

O interesse em estudar a exploração de um tipo de fractal surgiu pelo fato de que no estado do Paraná, no ano de 2008, as geometrias não euclidianas foram introduzidas nas Diretrizes Curriculares do Estado (DCE) tanto no Ensino Fundamental quanto no Ensino Médio. As DCE (PARANÁ, 2008) sugerem que no 8o ano o conteúdo estruturante de Geometrias seja trabalhado de forma que o aluno "[...] conheça os fractais através da visualização e manipulação de materiais e discuta suas propriedades" (PARANÁ, p.79, 2008) e que, no Ensino Médio, "Conheça os conceitos básicos da Geometria Elíptica, Hiperbólica e Fractal [...]" (PARANÁ, p.81, 2008).

Esta inclusão nos levou ao seguinte questionamento: será que os professores de Matemática da Educação Básica abordam a Geometria Fractal nas aulas de Matemática de modo a contemplar diferentes conteúdos e suas diferentes representações? Tal questionamento nos motivou a investigar um grupo de professores que ensinam matemática, participantes do Programa de Desenvolvimento Educacional (PDE)4. Ao refletir sobre os

${ }^{4} \mathrm{O}$ PDE compõe uma política pública do Estado do Paraná que visa o diálogo entre os professores do Ensino Superior com os professores da Educação Básica. Essa relação ocorre por meio de atividades teórico-práticas e 
primeiros passos da pesquisa, a pergunta inicial se desdobrou nos seguintes questionamentos: os professores da Educação Básica exploram diferentes Registros de Representação Semiótica ao elaborarem atividades que envolvam a Geometria Fractal? Se sim, o processo de conversão entre estas representações ocorre? De que maneira?

Desta forma, solicitamos aos professores participantes que respondessem a um questionário previamente elaborado que investigou, sob o ponto de vista desses professores: i) quais conteúdos podem ser explorados com este fractal; ii) atividades que podem ser aplicadas utilizando o Hexágono de Dürer; iii) relações matemáticas que podem ser estabelecidas entre as etapas de construção do fractal.

Assim, diante destas perguntas, o objetivo principal desta pesquisa foi identificar se nas atividades matemáticas elaboradas pelos professores, com base no Hexágono de Dürer, houve a manifestação dos diferentes tipos de Registros de Representação Semiótica (RRS) e como se deu a conversão entre eles. Para alcançar tal objetivo, identificamos quais registros tiveram maior e menor frequência de mobilização pelos professores durante a proposta de atividade elaborada. Além disso, observamos quais conversões podem ser exploradas, a partir dos dados coletados.

Na escrita deste trabalho será abordado primeiramente uma revisão bibliográfica a respeito da teoria dos RRS, em seguida descrevemos a respeito do uso do software GeoGebra em aulas de matemática e, para finalizar, uma breve introdução sobre a Geometria Fractal. Posteriormente, apresentamos uma análise a respeito de atividades matemáticas que foram elaboradas pelos sujeitos da pesquisa.

\section{As contribuições da Teoria dos Registros de Representação Semiótica}

Para Duval (2012, p.269), “As representações semióticas são produções constituídas pelo emprego de signos pertencentes a um sistema de representações que tem inconvenientes próprios de significação e de funcionamento". As representações semióticas utilizam sistemas semióticos diferentes e desempenham um papel importante tanto para fins de comunicação quanto para a formação do pensamento cognitivo (DUVAL, 2012).

Com relação às representações e à Matemática, Moretti (2002, p.344) afirma que "Em matemática, recorre-se a uma grande variedade de representações semióticas [...]", como exemplo, citamos as representações em língua natural, a escrita algébrica, gráficos e figuras geométricas. Segundo o mesmo autor, uma razão para a existência dessa grande variedade de representações deve-se ao fato de que, na aprendizagem, para Piaget e Vygotsky, as representações desempenham um papel decisivo. Silva, Alves e Noronha (2018), apresentaram resultados de uma pesquisa em que, por meio dos registros escritos dos alunos participantes, constatou-se a dificuldade de efetuar transformações de um registro a outro, e o cerne dessa dificuldade se encontrava no tratar e interpretar os dados da tarefa.

Duval, em entrevista concedida a Freitas e Rezende (2013), menciona que a matemática possui um caráter cognitivo e epistemológico específico. Segundo o pesquisador, “[...] não existe acesso perceptivo, direto ou instrumental (microscópio, telescópio, osciloscópio, espectroscópio, etc.) aos números, às funções, às relações geométricas, ou seja, aos objetos matemáticos" (p.16). Desse modo, para que se tenha acesso a esses objetos, é preciso uma atividade de produção semiótica.

o objetivo do programa é alcançar a produção de conhecimento e mudanças na prática escolar dos professores nas escolas públicas paranaenses. 
Desse modo, Duval (2012) defende que na atividade cognitiva matemática é essencial que se mobilizem diferentes registros de representação semiótica: figuras, gráficos, escritas simbólicas, língua natural e que se possa escolher entre um registro e outro. Neste sentido, com relação ao pensamento cognitivo matemático dos alunos, acreditamos, assim como Duval, que as diferentes representações semióticas contribuem para seu desenvolvimento e para a aprendizagem dos conceitos em questão.

Duval (2012) ressalta sobre a importância de os objetos e conceitos matemáticos não serem confundidos com suas representações, e sim que estes possam ser reconhecidos por meio de suas representações. A conversão entre as diferentes representações implicará, então, no entendimento dos objetos e conceitos matemáticos. Sobre a diferença entre as representações, Duval (1999) afirma que:

\begin{abstract}
As representações diferentes de um mesmo objeto, não têm evidentemente o mesmo conteúdo. Cada conteúdo é comandado por um sistema pelo qual a representação foi produzida. Daí a consequência de que cada representação não apresenta as mesmas propriedades ou as mesmas caraterísticas do objeto. Nenhum sistema de representação pode produzir uma representação cujo conteúdo seja completo e adequado ao objeto representado. (DUVAL 1999, p. 18).
\end{abstract}

Este pensamento de Duval nos explica que não existe um único tipo de representação que seja suficiente a ponto de proporcionar a melhor compreensão com relação a um conceito matemático. Para exemplificar, apresentamos uma situação referente às diferentes representações para uma mesma parábola. Para o registro simbólico-algébrico, com pequenas modificações (que Duval denomina de tratamento), Moretti (2002) apresenta três possibilidades de representação para uma mesma equação de parábola: 1) $y=x^{2}-4 x+3$; 2) $y+1=(x-2)^{2}$; 3$)(x-3)(x-1)$. O autor destaca que cada uma destas representações desperta o olhar para determinados elementos da parábola. A primeira equação permite identificar o ponto de intersecção com o eixo y; a segunda equação permite identificar quase que de imediato seu vértice $\mathrm{V}(2,-1)$; e a terceira equação permite identificar suas raízes. Além destas, uma outra representação e que se refere à mesma parábola diz respeito ao registro gráfico, que permite outro tipo de visualização do mesmo objeto matemático.

Quando transformamos uma representação em outro registro, de modo a conservar totalmente ou somente uma parte da representação inicial, realizamos uma conversão. Segundo Duval (2012), existem diferentes tipos de conversões, por exemplo: a ilustração, que é a conversão do registro língua natural para o registro figural, a tradução, que consiste na conversão do registro língua natural para outro tipo de língua e a descrição, que é a conversão do registro figural para o registro língua natural.

Duval (2012) ainda ressalta que é importante não haver confusão entre os termos conversão e tratamento, pois o tratamento de uma representação é a transformação desta no mesmo registro em que ela foi criada, ou seja, é uma transformação interna ao registro inicial. Por exemplo, o número 0,2 se encontra em uma representação numérica do registro simbólico e pode ser tratado como $\frac{2}{10}$ ou $2.10^{-1}$, embora esteja, no primeiro caso, em uma representação fracionária e no segundo caso na sua representação em forma de potência, apresentando significações operatórias diferentes (DUVAL, 1999). No entanto, este número pode ser convertido do registro simbólico para o registro da língua natural - dois décimos. "A 
conversão é uma atividade cognitiva diferente e independente do tratamento" (DUVAL, 2012, p.272).

Duval (2013) menciona sobre a existência de duas faces da atividade matemática, a face exposta e a face oculta. Como face exposta, o pesquisador define como sendo aquela que "[...] corresponde aos objetos matemáticos (números, funções, equações, polígonos, poliedros, etc.), às suas propriedades, às fórmulas e algoritmos aos quais eles dão origem, às demonstrações" (p.17). Ou seja, faz parte da face exposta os conteúdos e escolhas institucionais que os alunos devem adquirir ao término do período escolar. Como face oculta, Duval (2013) considera aqueles elementos que não estão direta e imediatamente perceptíveis em relação ao que observamos do trabalho dos alunos em sala de aula.

Tanto a face exposta quanto a face oculta da atividade matemática cabem ao professor cumpri-las em sala de aula. Porém, a face exposta, na maioria das vezes, é familiar ao professor, que tem ciência que no final do ano letivo precisa cumprir determinada programação, em conformidade com o currículo prescrito. Enquanto a face oculta, tão importante quanto a face exposta, raramente é cobrada pelo sistema de ensino, e cabe ao professor reconhecer a sua importância e incorporá-la em sua prática docente.

Nesse sentido, e considerando a atividade de conversão como um dos elementos presentes na face oculta da matemática, acreditamos que se a solicitação da conversão de diferentes representações semióticas não for proposta pelo docente, dificilmente será realizada pelo aluno. Nesse sentido, Duval (2012) afirma que:

Em matemática, mais do que em qualquer outra disciplina, estas mudanças são, ao mesmo tempo, importantes e frequentes. Basta abrir qualquer manual escolar de qualquer nível para ver, em uma mesma página, a passagem de uma frase para uma fórmula aritmética ou algébrica, a passagem de um enunciado para uma figura geométrica, ou a passagem da escrita algébrica para o gráfico. (DUVAL, 2012, p. 99).

No entanto, é necessária uma preocupação constante do professor em oportunizar atividades em que os alunos perpassem pelas diferentes representações semióticas até que tal ação seja realizada espontaneamente.

\section{0 software GeoGebra e a Geometria Fractal no ensino de Matemática}

A Geometria Fractal é caracterizada, dentre outras coisas, por sua autossimilaridade, uma propriedade especial de suas formas geométricas (fractais), que caracteriza que suas partes são semelhantes. Segundo Barbosa (2005), podemos utilizar essa geometria para modelar uma natureza irregular, pois ela oferece aproximações para essas formas. Benoit Mandelbrot foi o pioneiro em estudá-la e, para ele, a Geometria Fractal reflete uma natureza de irregularidades e fragmentações, e é uma forma cujas partes se assemelham ao seu todo sob alguns aspectos (BARBOSA, 2005). Como exemplo, temos o fractal Hexágono de Dürer da Figura 1, a seguir: 
Figura 1 - Fractal Hexagonal Tipo Dürer - Etapas

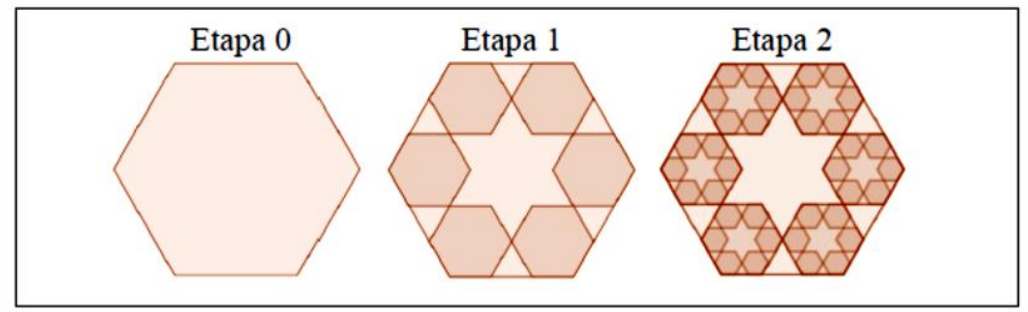

Fonte: Autoras

É possível observar nesse fractal da Figura 1, construído por meio do software GeoGebra, a presença da autossimilaridade infinita, já que novos e semelhantes hexágonos vão surgindo a cada etapa de construção. De acordo com Pimentel e Urban (2003), o fato de os fractais terem um padrão que se repete indefinidamente gera complexas figuras que preservam a singularidade da propriedade de que cada parte representa o todo.

O estudo do fractal geométrico proporciona também a investigação de diferentes conceitos matemáticos e não somente uma exploração figural. Ou seja, é possível investigar cada etapa de construção do fractal observando o comportamento deste objeto no que diz respeito ao seu perímetro, sua área, seus ângulos, as simetrias compostas, e dessa forma explorar aspectos de grandezas e medidas, conforme abordado na oficina proposta por esta pesquisa, fato que será explanado posteriormente.

Barbosa (2005) incentiva o trabalho com a Geometria Fractal e afirma:

Cremos, no entanto, que para os fractais, em especial para a geometria fractal, faz-se necessário ao educador conseguir captar o educando com o transparecer de sua própria vibração e talvez evidenciando o êxtase na contemplação da beleza de seus visuais, conduzindo-o ao prazer pelas informações e conhecimentos culturais da vasta variedade de fractais. (BARBOSA, 2005 p. 14).

Com relação a inclusão do conteúdo de geometrias não euclidianas no estado do Paraná, Bagio e Rolkouski (2014, p. 8-10) expõem:

Com vistas a tratar de assuntos relativos à implementação das DCE e dos novos conteúdos, foi criado em 2007 o DEB-Itinerante, [...] A proposta do DEB-Itinerante, para capacitar os professores se concretizava por meio de oficinas. [...] No caso das oficinas da área de Matemática, o conteúdo específico abordado foi o conteúdo de Geometrias Não Euclidianas.

Em uma pesquisa a respeito das produções paranaenses sobre a inclusão do ensino das geometrias não euclidianas nas DCE do Estado do Paraná, Bagio (2015, p. 18) afirma:

Em síntese, os resultados das pesquisas realizadas com relação às geometrias não euclidianas no Paraná podem ser divididos em três categorias gerais: i. A inserção desse conteúdo não foi algo simples e de concordância de todos os professores [...] ii. A formação inicial e continuada do professor de Matemática deveria preparar melhor a todos para o ensino adequado deste 
conteúdo [...] iii. A necessidade de mais materiais didáticos para o ensino desses conteúdos além dos propostos pela $\operatorname{SEED}^{5}[\ldots]$.

Bagio (2015) ainda destaca que conforme as pesquisas sobre o tema, os professores de Matemática, tanto na formação inicial quanto na continuada, ainda não se encontram preparados adequadamente para o ensino das geometrias não euclidianas. Acreditamos que este processo de preparação demanda tempo e não é algo simples de se realizar, pois os cursos de formação inicial de professores que ensinam matemática também necessitam readequar suas matrizes curriculares para suprir tal necessidade.

O uso do software GeoGebra em cursos de formação de professores, é uma boa alternativa para essa readequação. "Embora as discussões em torno de educação e tecnologia não sejam recentes, as tecnologias digitais ainda não foram incorporadas às práticas profissionais de grande parte dos professores" (BASNIAK; SILVA, 2018, p. 169). Com relação a isso, Cyrino e Baldini (2012, p. 59-60) relatam que:

[...] nos cursos de formação de professores (inicial e continuada), ao trabalhar com o uso das TIC, podem ser oferecidos momentos nos quais eles tenham a oportunidade de: analisar um software como uma ferramenta que permite ao aluno construir novos conhecimentos matemáticos; [...] investigar diferentes formas de utilizar o software para dinamizar as aulas, promovendo a interação entre o aluno e o objeto matemático; [...] elaborar propostas alternativas de organização e gestão dos processos de ensino e de aprendizagem, por meio do uso do software, de modo a valorizar as interações entre os alunos, entre o aluno e o professor; [...] analisar e reconhecer diferentes formas de pensamento e de registros, provocadas pelo uso do software [...].

Cury e Leivas (2008) acreditam que gerar fractais utilizando o software GeoGebra em trabalhos com professores de formação continuada pode proporcionar a estes a aquisição de conhecimentos que não tiveram oportunidade de conhecer em sua formação inicial. Faria e Maltempi (2012) afirmam que o software GeoGebra proporciona a exploração de um mesmo fractal em diferentes níveis. Estes autores ainda ressaltam que o software é capaz de estimular o teste de algumas conjecturas proporcionando assim a comprovação ou refutação de algumas ideias. Sendo assim, o software Geogebra permite verificar, dentre outras coisas, o comportamento (padrão) de um fractal em $n$ etapas, por exemplo.

Baseadas nestes estudos, realizamos a investigação da construção do Fractal Hexagonal tipo Dürer, utilizando o software GeoGebra com professores em formação continuada, membros do PDE, com o intuito de conscientizá-los de que esta geometria, quando bem explorada, pode auxiliar no processo de ensino e aprendizagem de diferentes conteúdos matemáticos. O professor é o principal mediador entre o aluno e o conhecimento, por isso o interesse em trabalhar com estes sujeitos de pesquisa e utilizando esta metodologia. Na seção a seguir descreveremos como se deu o processo de investigação.

\section{Descrição e análises da investigação}

O presente trabalho tem sua natureza metodológica pautada sobre a pesquisa qualitativa, uma vez que pretende interpretar os fenômenos manifestados pelos seus

${ }^{5}$ SEED - Secretaria de Estado da Educação do Paraná. 
participantes, conforme indicam Denzin e Lincoln (2011). Neste sentido, para dar andamento na proposta e realizar a coleta de dados, propusemos um curso, com duração de 4 horas, para um grupo de 14 professores do PDE, que visou a construção do fractal Hexagonal Tipo Dürer com o auxílio do software GeoGebra, e ocorreu em agosto de 2016, no Laboratório de Informática da Universidade Estadual do Paraná - UNESPAR, Campus de Campo Mourão. 0 tempo destinado para a construção e preenchimento das questões foi de aproximadamente 4 horas. Para a análise dos dados, denotamos os participantes por P1, P2, P3, assim por diante, até $\mathrm{P} 14$, para preservar as suas identidades. Os professores foram informados de que o minicurso serviria como um meio para uma investigação, no entanto o foco da pesquisa não foi mencionado para evitar influências na coleta dos dados.

Para realizar a construção do fractal, solicitamos que os professores se agrupassem em duplas e, para melhor instruí-los, com o auxílio de um projetor multimídia, projetamos a imagem do software GeoGebra na lousa. Os passos da construção foram relatados oralmente por uma das pesquisadoras e, simultaneamente, o fractal foi construído passo a passo. Em caso de dúvidas com relação à construção, retomávamos os passos ou instruíamos cada dupla de professores. Elaboramos um tutorial para orientar a construção do fractal que foi utilizado pelos pesquisadores no momento da implementação. A Figura 2, a seguir, apresenta o fractal construído até a terceira etapa.

Figura 2 - Fractal Hexagonal Tipo Dürer - Etapa 3

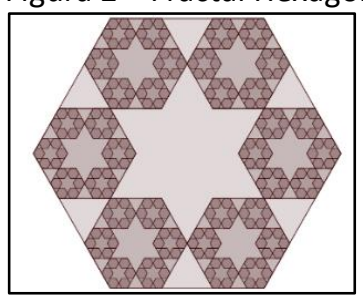

Fonte: Autoras

A construção do fractal, realizada com o auxílio do software GeoGebra, se desenvolveu nos seguintes passos: instruímos os professores a construir um hexágono inicial. Feito isto instruímos a construir 6 hexágonos no interior deste (inicial), já que o fractal Hexagonal Tipo Dürer é caracterizado por possuir 6 novos hexágonos, no interior de cada hexágono já existente, renovando-se esse processo a cada etapa. Por fim, foi criada uma ferramenta no software GeoGebra, a qual otimizava o tempo de construção de cada etapa.

Após realizarmos a construção do fractal, distribuímos um questionário para os professores responderem individualmente. No momento da implementação do questionário procuramos não influenciar nas respostas dos professores. A seguir, apresentamos as análises de cada questão e os resultados alcançados.

4.1 Análise da questão 1: Ao elaborarmos a questão 1: "Quais conteúdos matemáticos você considera possível explorarmos durante e após a construção do Fractal Hexagonal Tipo Dürer?", tivemos por objetivo identificar quais conteúdos específicos seriam citados por cada um dos professores e desse modo enquadrá-los nos conteúdos estruturantes 6 descritos nas DCE - Diretrizes Curriculares do Estado do Paraná (PARANÁ, 2008). Tem-se nas DCE, como proposta para a Educação Básica da rede pública estadual, os seguintes conteúdos

\footnotetext{
6 "Entende-se por conteúdos estruturantes os conhecimentos de grande amplitude, conceitos, teorias ou práticas, que identificam e organizam os campos de estudos de uma disciplina escolar, considerados fundamentais para a compreensão de seu objeto de estudo/ensino [...] Dos conteúdos estruturantes organizamse os conteúdos básicos a serem trabalhados por série [...]" (PARANÁ, p.23-26, 2008).
} 
estruturantes: Números e Álgebra; Grandezas e Medidas; Geometrias; Funções e Tratamento da Informação (PARANÁ, 2008). Assim, com base no que é indicado por conteúdo estruturante, os quais englobam os conteúdos básicos, analisamos as respostas apresentadas para a questão 1. No Quadro 1, apresentamos o levantamento dos conteúdos estruturantes abordados com base nas respostas dos professores participantes.

Quadro 1 - Conteúdos estruturantes citados como resposta para a questão 1

\begin{tabular}{|c|c|}
\hline CONTEÚDO ESTRUTURANTE & PROFESSOR \\
\hline Funções & P1 e P2 \\
\hline Geometrias & P1, P2, P3, P4, P5, P6, P7, P8, P9, P10, P11, \\
& P12, P13 e P14 \\
\hline Grandezas e Medidas & P1, P2, P3, P4, P5, P6, P8, P12, P13 e P14 \\
\hline Números e Álgebra & P7, P8, P9, P10, P11, P13 e P14 \\
\hline
\end{tabular}

Fonte: Autoras

Como pode-se observar, o conteúdo mencionado com maior frequência foi o de Geometrias, enquanto o conteúdo de Funções foi o menos citado. Já o conteúdo Tratamento da Informação não foi mencionado na resposta de nenhum professor. As DCE indicam que, no Ensino Médio, o conteúdo estruturante de Tratamento da Informação aborde algumas noções de Probabilidade. No artigo intitulado "O ensino de probabilidade geométrica por meio de fractais e da resolução de problemas" 7 , Lopes et al (2013) apresentam exemplos de atividades que focam nas noções de probabilidade por meio de alguns fractais. Neste contexto, acreditamos que alguns conceitos de Probabilidade podem ser trabalhados por meio do Hexágono de Dürer, abarcando, assim, parte do conteúdo de Tratamento da Informação.

Com relação ao conteúdo estruturante de Funções para o Ensino Médio, pode-se observar a presença deste, por exemplo, na resposta apresentada pelo professor P1 ao citar: Sequências Numéricas, Progressão Geométrica e Funções. As DCE indicam que para este nível o aluno "[...] reconheça, nas sequências numéricas, particularidades que remetam ao conceito das progressões aritméticas e geométricas; [...]" (PARANÁ, p. 81, 2008). A figura a seguir apresenta a resposta do professor $\mathrm{P} 1$. Além disso, também consideramos que o conceito de função pode ser explorado por meio das generalizações estabelecidas pelas etapas e áreas ou perímetros do fractal de Dürer, por exemplo.

Figura 3 - Respostas da questão 1 apresentada pelo professor P1.

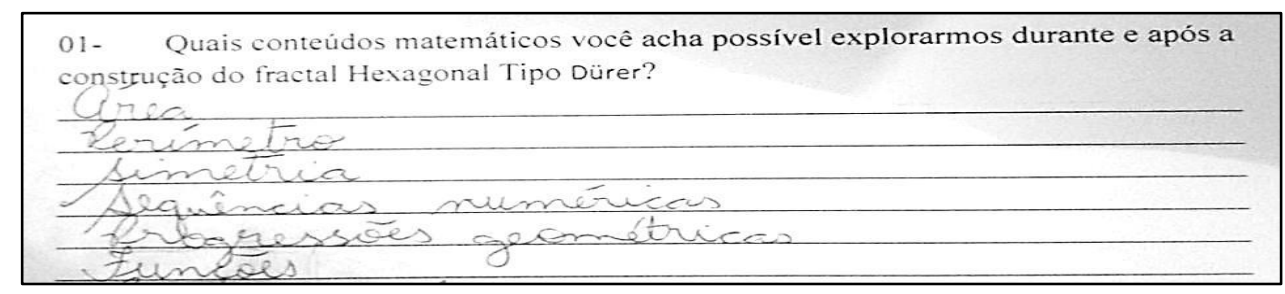

Fonte: Autoras

As DCE indicam que no Ensino Fundamental o conteúdo estruturante de Geometrias seja trabalhado de modo que "[...] o aluno deve compreender: os conceitos da geometria plana: ponto, reta e plano; [...] cálculos geométricos: perímetro e área [...]" (PARANÁ, p.56,

${ }^{7}$ Disponível em: http://www.reveduc.ufscar.br/index.php/reveduc/article/view/500 
2008). Com relação ao Ensino Médio, os alunos devem realizar: "[...] análises dos elementos que estruturam a geometria euclidiana. [...] Também, no Ensino Médio, aprofundam-se os estudos das noções de geometrias não euclidianas ao abordar a geometria dos fractais [...] (PARANÁ, p. 57, 2008).

Assim, identificamos nas respostas apresentadas, por exemplo, pelos P3 e P4, a presença do conteúdo estruturante de geometria para ser trabalhado no Ensino Fundamental, pois estes citam geometria plana e plano cartesiano. Já na resposta apresentada pelo P14, identificamos a presença deste conteúdo para um trabalho tanto no Ensino Fundamental quanto no Ensino Médio, pois este professor cita perímetro de fractais.

Para um trabalho com o Ensino Fundamental, com relação ao conteúdo estruturante de Grandezas e Medidas, as DCE sugerem que este seja trabalhado os conceitos de áreas e volumes, medidas de ângulos, entre outros. Assim, com base no que é indicado pelas DCE, identificamos a presença do conteúdo de Grandezas e Medidas na resposta apresentada pelo P12, ao mencionar: ângulos e área, dentre outros conceitos desse conteúdo estruturante, eu sua resposta.

Com relação ao conteúdo Números e Álgebra para o Ensino Fundamental, as DCE sugerem que este seja trabalhado de forma que os alunos compreendam: "[...] o conceito de razão e proporção, regra de três, porcentagem, frações e dos números decimais e as suas operações" (PARANÁ, p.51, 2008). Assim, identificamos a presença deste conteúdo na resposta do P10 ao citar razão e proporção, e na resposta apresentada pelo P14 ao citar frações. A seguir as Figuras 4 e 5 apresentam a respostas destes professores.

Figura 4 - Respostas da questão 1 apresentada pelo professor P10.

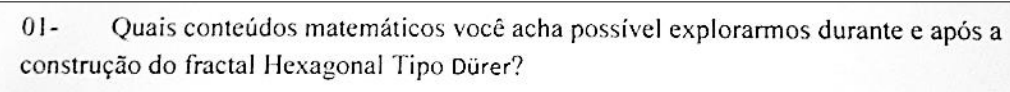

Fonte: Autoras

Figura 5 - Respostas da questão 1 apresentada pelo professor P14.

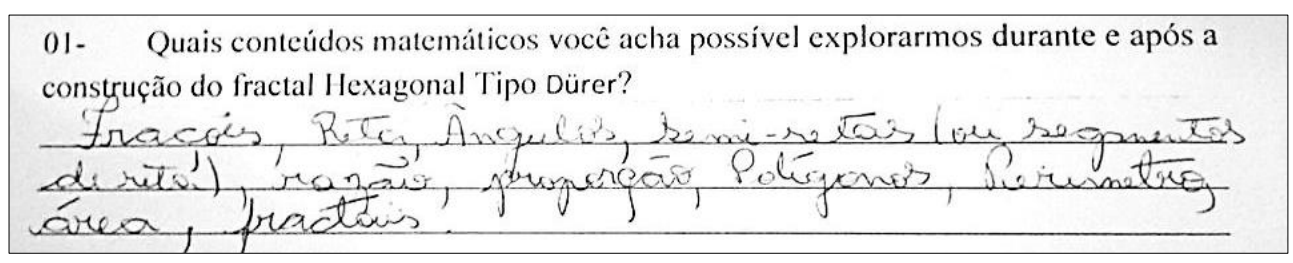

Fonte: Autoras

Após a análise da questão 1, observamos que todos os professores participantes identificaram a possibilidade de explorar conteúdos matemáticos, além das geometrias, durante e após a construção do fractal proposto. Além disso, pelo fato de identificarmos nas respostas conteúdos estruturantes de diferentes níveis de ensino, ressaltamos que a construção do fractal com o auxílio do software GeoGebra proporciona a exploração de conteúdos matemáticos com alunos em diferentes faixas etárias, e, com base nas respostas disponibilizadas no Quadro 1, observamos que todos os sujeitos colaboradores desta pesquisa têm consciência e visibilidade desta opção, o que contribui para os processos de ensino e aprendizagem da matemática, além de proporcionar aos alunos o conhecimento e a apreciação do belo existente na Geometria Fractal. 
4.2 Análise da Questão 2: Ao propormos a questão 2: "Elabore uma atividade que contenha pelo menos três questões (itens), a qual você aplicaria em sala de aula para explorar os conteúdos listados na questão 1. Relate quais suas expectativas em cada item elaborado, com relação à aprendizagem dos alunos", tivemos por objetivo identificar quais registros de representação semiótica: simbólico (RS) podendo ser numérico (RSN) e/ou algébrico (RSA), língua natural (RLN) e/ou figural (RF), os professores solicitariam que os alunos reproduzissem durante a resolução da atividade elaborada por eles. Vale ressaltar que o fractal Hexagonal Tipo Dürer, representado aos professores por meio do software Geogebra, é considerado uma representação pertencente ao registro figural, assim as possibilidades de conversões que podem surgir no decorrer da resolução da atividade elaborada, consequentemente, realizarse-iam do RF para os outros já mencionados.

Durante a análise percebemos que alguns enunciados elaborados pelos professores não ficaram claros ou coerentes. Além disso, esclarecemos que não foi solicitado que as atividades elaboradas fossem resolvidas por nenhum aluno ou professor.

Nessas condições, identificamos nas análises da questão 2, as representações semióticas manifestadas pelos professores. As tabelas a seguir apresentam as questões contidas nas atividades elaboradas por cada um dos professores, as representações semióticas solicitadas em cada uma e as possibilidades de conversões a serem realizadas pelo aluno. No decorrer do texto descrevemos as questões de cada atividade, sem modificações, feitas pelos professores.

Quadro 2 - Análise da atividade elaborada pelo professor P1.

\begin{tabular}{|l|c|c|}
\hline \multicolumn{1}{|c|}{ Atividade } & Registro & Conversão \\
\hline $\begin{array}{c}\text { a) Construa uma tabela relacionando a quantidade de hexágonos } \\
\text { (peças) dentro de um hexágono (maior) }\end{array}$ & $\mathrm{RSN}$ & $\mathrm{RF} \rightarrow \mathrm{RSN}$ \\
\hline b) Relacionar os dados da tabela com o no de lados do polígono & $\mathrm{RSN}$ & $\mathrm{RF} \rightarrow \mathrm{RSN}$ \\
\hline c) Relacionar a área de cada hexágono encontrado, perímetro, etc. & $\mathrm{RSN}$ & $\mathrm{RF} \rightarrow \mathrm{RSN}$ \\
\hline
\end{tabular}

Fonte: Autoras

Ao analisarmos a atividade elaborada pelo professor $\mathrm{P} 1$, identificamos que em todos os itens solicitou-se o uso do RS na forma de representação numérica. Especificamente para o item c), pelo fato de o professor não explicitar uma medida para o lado do hexágono inicial, acreditamos que o aluno consideraria a medida que foi estabelecida para a construção, realizada com o auxílio do software GeoGebra. Portanto, nas três questões, a possibilidade de conversão será do RF para o RS.

Quadro 3 - Análise da atividade elaborada pelo professor P2.

\begin{tabular}{|c|c|c|}
\hline Atividade & Registro & Conversão \\
\hline $\begin{array}{c}\text { a) Medir os lados dos hexágonos maiores e menores e verificar se há } \\
\text { proporcionalidade entre eles. }\end{array}$ & RSN & RF $\rightarrow$ RSN \\
\hline
\end{tabular}

Fonte: Autoras

Solicitamos que na questão 2 fosse elaborada uma atividade que contemplasse pelo menos três itens (questões), e que nestes fossem explorados os conteúdos listados na questão 1 , porém houve caso de professores que elaboraram apenas um único item em sua atividade, como foi o caso do professor P2. Na atividade elaborada por ele, identificamos a solicitação 
do RS. Percebe-se que neste item o objetivo foi trabalhar com proporcionalidade porém, este tema não foi mencionado por este professor em sua resposta para a questão 1.

Quadro 4-Análise da atividade elaborada pelo professor P3.

\begin{tabular}{|l|c|c|}
\hline \multicolumn{1}{|c|}{ Atividade } & Registro & Conversão \\
\hline $\begin{array}{l}\text { a) Criação de pontos para bordado nas roupas ucranianas ponto } \\
\text { forte de cultura na região (cidade). }\end{array}$ & RF & $\begin{array}{c}\text { Não } \\
\text { solicitada }\end{array}$ \\
\hline $\begin{array}{l}\text { b) Construção de modelos para calota de carros. Cálculo de gastos } \\
\text { de material. }\end{array}$ & RSN & RF $\rightarrow$ RSN \\
\hline
\end{tabular}

Fonte: Autoras

O professor P3 elaborou uma atividade que explorou um pouco de desenho geométrico ao solicitar a construção de modelos para calota de carros e, consequentemente, solicitou-se a conversão do registro figural para o simbólico na representação numérica quando designou o cálculo de gastos de material.

Quadro 5 - Análise da atividade elaborada pelo professor P4.

\begin{tabular}{|l|c|c|}
\hline \multicolumn{1}{|c|}{ Atividade } & Registro & Conversão \\
\hline $\begin{array}{l}\text { a) Definir: polígono, polígono regular, nomear a figura de acordo com } \\
\text { os lados, identificar ângulos e medir. }\end{array}$ & RLN e RSN & $R F \rightarrow$ RLN \\
\cline { 3 - 3 } & & $R F \rightarrow$ RSN \\
\hline
\end{tabular}

Fonte: Autoras

O professor P4 apresentou mais um caso de atividade elaborada com somente um item, tal fato nos leva a refletir a respeito das poucas informações disponíveis em seu repertório para que pudesse elaborar outras atividades. Identificamos a solicitação do registro língua natural, pois o professor menciona as palavras "definir e nomear" e também a solicitação do registro numérico, já que o professor cita a palavra "medir". Consequentemente, as possibilidades de conversão serão do RF para o RLN e do RF para o RS, este último na forma de representação numérica.

Quadro 6-Análise da atividade elaborada pelo professor P5.

\begin{tabular}{|l|c|c|}
\hline \multicolumn{1}{|c|}{ Atividade } & Registro & Conversão \\
\hline $\begin{array}{l}\text { a) No decorrer do trabalho procura-se apresentar diferentes formas } \\
\text { de fractais. Podemos a partir dos fractais construir novos fractais, } \\
\text { mantendo a semelhança. }\end{array}$ & RF & Não solicitada \\
\hline
\end{tabular}

Fonte: Autoras

$\mathrm{O}$ professor $\mathrm{P} 5$ também elaborou apenas um item em sua atividade, no entanto ao invés de solicitar uma conversão entre registros, esse professor permaneceu no registro figural caracterizando a atividade como uma proposta de tratamento figural.

Quadro 7 - Análise da atividade elaborada pelo professor P6.

\begin{tabular}{|l|c|c|}
\hline \multicolumn{1}{|c|}{ Atividade } & Registro & Conversão \\
\hline $\begin{array}{l}\text { a) Sendo o lado de um quadrado A e B, marque os outros pontos e } \\
\text { determine os outros lados deste quadrado. }\end{array}$ & RF & Não solicitada \\
\hline $\begin{array}{l}\text { b) Obs: Em quais segmentos você teve que marcar com o mouse para } \\
\text { obter os outros segmentos? }\end{array}$ & Não houve & Não solicitada \\
\hline
\end{tabular}

Fonte: Autoras

As figuras que surgem em cada etapa do fractal Hexagonal Tipo Dürer apresentam formato de um hexágono que contém explicitamente triângulos equiláteros. Logo, o item a) 
elaborado na atividade pelo professor P6 não se relaciona diretamente com a construção do fractal Hexagonal Tipo Dürer. Consequentemente, o item b), que está relacionado como item a), também não apresentou relações com a construção do fractal. O professor $P 6$ relatou que "Esta atividade pode ser feita com outras figuras com maior número de lados para os alunos observarem a formação dos lados e dos vértices, para o 60 ano"!

Quadro 8-Continuação da análise da atividade elaborada pelo professor P7.

\begin{tabular}{|c|c|c|c|}
\hline & Atividade & Registro & Conversão \\
\hline & $\begin{array}{l}\text { Sendo o lado do hexágono } x \text {, determine o perímetro em cada } \\
\text { uma das evoluções. }\end{array}$ & $\mathrm{RS}$ & $\mathrm{RF} \rightarrow \mathrm{RSA}$ \\
\hline b) & $\begin{array}{l}\text { Calcule a área de cada hexágono, sendo determinada a medida } \\
\text { do lado. }\end{array}$ & RS & $\mathrm{RF} \rightarrow \mathrm{RSN}$ \\
\hline c) & $\begin{array}{l}\text { Calcule a área de cada hexágono, sendo determinada a medida } \\
\text { do lado. }\end{array}$ & RS & $\mathrm{RF} \rightarrow \mathrm{RSN}$ \\
\hline d) & $\begin{array}{l}\text { Calcule a área do hexágono inicial, a área dos hexágonos na } 1^{\mathrm{a}} \\
\text { evolução e após a área não ocupada pelos hexágonos. }\end{array}$ & RS & $\mathrm{RF} \rightarrow \mathrm{RSN}$ \\
\hline & $\begin{array}{l}\text { Fazer relação entre as áreas em cada uma das evoluções, } \\
\text { comparando os resultados. }\end{array}$ & $\mathrm{RS}$ & $\mathrm{RF} \rightarrow \mathrm{RSN}$ \\
\hline
\end{tabular}

Fonte: Autoras

O professor P7 foi o único que solicitou em sua atividade o registro simbólico numa representação algébrica, conforme consta no item a). Com relação aos itens b) e c), identificase a presença do registro simbólico na forma numérica, e consequentemente, a conversão do RF para o RSN. Por fim, no item d), como o cálculo das áreas foi realizado numericamente, a relação entre os valores encontrados também será numérica, logo ocorrerá a presença do RSN e a possibilidade de conversão do RF para o RSN. Com os itens b), c) e d) objetivou-se explorar sobre área. No entanto, o item a) indicou encontrar uma relação algébrica para o perímetro. Estes temas foram listados como resposta pelo professor P7 para a questão 1.

Quadro 9-Análise da atividade elaborada pelo professor P8.

\begin{tabular}{|c|c|c|c|}
\hline & Atividade & Registro & Conversão \\
\hline a) & $\begin{array}{l}\text { Nomear os polígonos que aparecem na figura, dizendo se são } \\
\text { regulares ou não. }\end{array}$ & RLN & $\mathrm{RF} \rightarrow \mathrm{RLN}$ \\
\hline b) & $\begin{array}{l}\text { Nomear os triângulos segundo as medidas dos lados e também } \\
\text { dos ângulos. }\end{array}$ & RLN & $\mathrm{RF} \rightarrow \mathrm{RLN}$ \\
\hline c) & $\begin{array}{l}\text { Calcular o perímetro dos triângulos sabendo a medida do lado } \\
\text { do hexágono }\end{array}$ & RSN & $\mathrm{RF} \rightarrow \mathrm{RSN}$ \\
\hline
\end{tabular}

Fonte: Autoras

Analisando as questões propostas na atividade elaborada pelo professor P8, identificamos que, no item a) e no item b), ocorreu a solicitação do RLN e, consequentemente, a possibilidade de conversão do RF para o RLN, solicitando um tipo de conversão descritiva. Como afirma Duval (2012, p. 272), “A descrição é a conversão de uma representação não verbal (esquema, figura, gráfico) em uma função linguística". Com relação ao item c), identificou-se a presença do registro simbólico na forma numérica e, consequentemente, a conversão do RSN para o RLN.

$\mathrm{O}$ item a) pretendeu explorar sobre polígono regular, tema mencionado pelo professor P8 como resposta para a questão 1. A exploração sobre triângulos solicitada no item b), não 
foi mencionada na resposta do professor P8 para a questão 1. Já com o item c) pretendeu-se explorar sobre perímetro. O professor $\mathrm{P} 8$ relatou sua expectativa com relação a aprendizagem do aluno ao mencionar "Que os conteúdos abordados sejam compreendidos pelos alunos, sendo a base para novos conhecimentos e estudos".

Quadro 10 - Análise da atividade elaborada pelo professor P9.

\begin{tabular}{|l|c|c|}
\hline \multicolumn{1}{|c|}{ Atividade } & Registro & Conversão \\
\hline $\begin{array}{l}\text { a) } \begin{array}{l}\text { Calcule o perímetro do maior polígono sendo dado as medidas } \\
\text { dos seus lados. }\end{array} \\
\text { b) Nomear os polígonos que aparecem na figura. }\end{array}$ & $\mathrm{RF} \rightarrow \mathrm{RSN}$ \\
\hline $\begin{array}{l}\text { c) } \\
\text { Nomear os triângulos que aparecem na figura de acordo com os } \\
\text { tamanhos dos seus lados. }\end{array}$ & $\mathrm{RLN}$ & $\mathrm{RF} \rightarrow \mathrm{RLN}$ \\
\hline
\end{tabular}

Fonte: Autoras

$\mathrm{Na}$ atividade elaborada pelo professor $\mathrm{P9}$, identificamos que no item a) ocorreu a solicitação do RSN, e consequentemente a possibilidade de conversão do RF para o RSN. Com relação aos itens b) e c), identifica-se a presença do RLN e a conversão do RF para o RLN.

Com os itens a) e b) pretendeu-se explorar sobre perímetro, tema mencionado pelo professor P9 como resposta para a questão 1 . O item b) teve a intenção de explorar sobre triângulos, porém este tema não foi mencionado na resposta do professor P9 para a questão 1. Já o item c) pretendeu explorar sobre polígono regular, tema que também foi mencionado na resposta para a questão 1 . O professor P9 relatou sua expectativa com relação à aprendizagem do aluno ao mencionar "Que os conteúdos citados e abordados dessa maneira, sejam mais fáceis para os alunos compreenderem".

Quadro 11 - Análise da atividade elaborada pelo professor P10.

\begin{tabular}{|ll|c|c|}
\hline \multicolumn{1}{|c|}{ Atividade } & Registro & Conversão \\
\hline a) & Quantos eixos simétricos possui o hexágono? & $\mathrm{RF} \rightarrow \mathrm{RSN}$ \\
\hline b) & Calcule o perímetro de hexágono maior. & $\mathrm{RSN}$ & $\mathrm{RF} \rightarrow \mathrm{RSN}$ \\
\hline $\begin{array}{l}\text { Além dos hexágonos e triângulos existe mais algum } \\
\text { polígono? }\end{array}$ & $\mathrm{RLN}$ & $\mathrm{RF} \rightarrow \mathrm{RLN}$ \\
\hline
\end{tabular}

Fonte: Autoras

O professor P10, no item a) e no item b), solicitou o RSN e, então, pode-se perceber que o mesmo teve por objetivo explorar simetria e perímetro, tais temas foram mencionados em sua resposta para a questão 1 . No item c) o professor solicitou ao aluno que por meio das características do polígono fosse identificado o polígono pelo seu nome.

Sobre as expectativas relatadas pelo professor com as questões, foram mencionadas as seguintes: "Que o aluno entenda o que é simetria e use papel para demonstrar", "Se vai calcular o perímetro se confundir com a área", "Definir as formas geométricas".

Quadro 12 - Análise da atividade elaborada pelo professor P11.

\begin{tabular}{|c|c|c|c|}
\hline & Atividade & Registro & Conversão \\
\hline a) & Quais tipos de polígonos se formaram? & RLN & $\mathrm{RF} \rightarrow \mathrm{RLN}$ \\
\hline b) & $\begin{array}{l}\text { Estabeleça a razão entre o hexágono maior e o hexágono } \\
\text { menor com o uso de régua. }\end{array}$ & RSN & $\mathrm{RF} \rightarrow \mathrm{RSN}$ \\
\hline c) & $\begin{array}{l}\text { Com o uso de uma régua realize as medições e calcule o } \\
\text { perímetro e a área dos diferentes polígonos. }\end{array}$ & RSN & $\mathrm{RF} \rightarrow \mathrm{RSN}$ \\
\hline
\end{tabular}

Fonte: Autoras 
Ao elaborar o enunciado do item a), acreditamos que P11 teve a intenção que o aluno identificasse se o fractal Hexagonal Tipo Dürer poderia ser formado por outros polígonos além dos hexágonos. Assim, neste item foi solicitado o RLN e, consequentemente a conversão do RF para o RLN. A mudança do registro simbólico na forma numérica ocorreu nos itens b) e c). Com relação ao item c), ao ser mencionado "[...] realize as medições e calcule [...]", identificamos também a manifestação do RSN. Nos itens a) e b) as conversões realizadas ocorreram do RF para o RSN.

O objetivo de P11 com a elaboração destas questões, é trabalhar razão, área e perímetro, itens mencionados na resposta da questão 2 .

Quadro 13 - Análise da atividade elaborada pelo professor P12.

\begin{tabular}{|l|c|c|}
\hline \multicolumn{1}{|c|}{ Atividade } & Registro & Conversão \\
\hline $\begin{array}{l}\text { Desenhe um hexágono com medida 3 cm entre os pontos. } \\
\begin{array}{l}\text { Determine. } \\
\text { a) Seu perímetro }\end{array}\end{array}$ & $\begin{array}{r}\mathrm{RF} \\
\mathrm{RSN}\end{array}$ & \\
\hline b) Sua área & $\mathrm{RSN}$ & $\mathrm{RF} \rightarrow \mathrm{RSN}$ \\
\hline c) Qual a medida de cada ângulo do hexágono & $\mathrm{RSN}$ & $\mathrm{RF} \rightarrow \mathrm{RSN}$ \\
\hline
\end{tabular}

Fonte: Autoras

$\mathrm{Na}$ atividade elaborada por P12 ocorreu a intenção de mobilização do próprio registro figural e do registro simbólico na forma numérica, sem conexão com a estrutura de um fractal. Nos demais itens a atividade se referiu somente ao registro simbólico na forma numérica. 0 professor P12 teve por objetivo, ao elaborar estes itens, trabalhar perímetro, área e ângulos, itens mencionados em sua resposta para a questão 2 . Os professores $\mathrm{P} 11$ e $\mathrm{P} 12$, também não relataram suas expectativas com relação à aprendizagem do aluno.

Quadro 14 - Análise da atividade elaborada pelo professor P13.

\begin{tabular}{|l|c|c|}
\hline \multicolumn{1}{|c|}{ Atividade } & Registro & Conversão \\
\hline a) Escrever o nome do polígono formado & $\mathrm{RLN}$ & $\mathrm{RF} \rightarrow \mathrm{RLN}$ \\
\hline b) Escrever a razão de um polígono para o outro & $\mathrm{RSN}$ & $\mathrm{RF} \rightarrow \mathrm{RSN}$ \\
\hline c) Encontrar a área dos polígonos desejados & $\mathrm{RSN}$ & $\mathrm{RF} \rightarrow \mathrm{RSN}$ \\
\hline
\end{tabular}

Fonte: Autoras

A solicitação do RLN é identificada no item a) elaborado pelo professor P13, pois este solicita: "Escrever o nome do polígono formado". Já o RSN foi solicitado por este professor nos itens b) e c). As expectativas deste professor com relação aos itens elaborados foram: "Identificar a figura formada", "Estabelecer a razão entre as figuras" e "Calcular a área das figuras".

Quadro 15: Análise da atividade elaborada pelo professor P14.

\begin{tabular}{|l|c|c|}
\hline \multicolumn{1}{|c|}{ Atividade } & Registro & Conversão \\
\hline a) Identifique e nomeie: & RSA & RF $\rightarrow$ RLN \\
i) Um segmento de Reta; ii) Um ângulo; ii) Um ponto & & \\
\hline & & \\
\hline b) Escreva o número de lados que o polígono da figura possui e & $\mathrm{RSN}$ & $\mathrm{RF} \rightarrow \mathrm{RSN}$ \\
& $\mathrm{RLN}$ & $\mathrm{RF} \rightarrow \mathrm{RLN}$ \\
\hline
\end{tabular}


\begin{tabular}{|l|l|l|}
\hline c) Utilizando medidas represente uma razão entre dois polígonos & RSN & RF $\rightarrow$ RSN \\
\hline
\end{tabular} Fonte: Autoras

O professor P14 também contemplou na elaboração de sua atividade o indicativo de mais de um registro. Identificamos no item a) a presença do registro simbólico algébrico e no item b) a solicitação do RLN e do RSN. Já no item c) foi solicitado apenas o RSN. As conversões com relação a estas questões foram do RF para o RLN, RSN e RSA. O professor P14 relatou que suas expectativas com relação à aprendizagem do aluno ao elaborar as questões a), b) e c) foram, respectivamente: "Reconhecimento e identificação de um ponto, segmento de reta e ângulo", "Identifique a nomenclatura do polígono e o no de lados", "Compreenda as noções de medida em um polígono e a razão que pode ser representada através do fractal". Com relação às expectativas dos professores P1, P2, P3, P4 e P7, sobre a aprendizagem do aluno, estes não relataram quais seriam.

Após a análise da questão 2, identificamos que os registros de representação semiótica demandados pelos professores nas atividades elaboradas foram: simbólico numérico, simbólico algébrico e língua natural. A representação mais solicitada foi a numérica abordada em 22 itens. Com relação à representação algébrica, esta foi a menos solicitada nas atividades, sendo indicada por um professor em apenas 1 item. Por fim, identificamos que o registro língua natural foi solicitado em 9 itens das atividades elaboradas. O quadro 16 apresenta um levantamento da quantidade de questões que solicitaram alguma transformação de registro.

Apenas 7 professores solicitaram em suas atividades mais de um tipo de possibilidade conversão entre as representações semióticas. Três professores não requisitaram a conversão entre registros e o restante dos professores solicitou apenas um tipo de conversão. Conforme mostra o quadro a seguir:

Quadro 16 - Análise das conversões indicadas pelos professores.

\begin{tabular}{|c|c|}
\hline Participantes & Conversão \\
\hline P4, P7, P8, P9, P11, P13, 14 & $\begin{array}{c}\text { RF } \rightarrow \text { RLN } \\
\text { RF } \rightarrow \text { RS }\end{array}$ \\
\hline P1, P2, P10, P12 & RF $\rightarrow$ RS \\
\hline P3, P5, P6 & Nenhuma \\
\hline
\end{tabular}

Fonte: Autoras

Como podemos observar no Quadro 16, os professores P1, P2, P10 e P12 propuseram apenas a conversão do RF para o RS. Já os P4, P8, P9, P11, P13 e P14 pensaram na conversão do RF para o RLN e do RF para o RS. Os professores P3, P5 e P6 não manifestaram nenhum tipo de conversão entre registros durante suas atividades.

Portanto, com base nas atividades elaboradas pelos professores observamos que 7 professores, de um total de 14, tiveram consciência da possibilidade de trabalho com ao menos três diferentes representações, e suas mobilizações, mesmo sem terem conhecimento da teoria dos registros de representação semiótica.

4.3 Análise da questão 3: Com relação à terceira questão do questionário, tivemos por objetivo verificar se os professores conseguiam estabelecer alguma relação entre a medida da área ou do perímetro dos novos hexágonos que surgem em cada etapa do fractal Hexagonal 
Tipo Dürer. Caso estabelecida alguma relação, buscamos analisar qual tipo de registro semiótico - figural ou simbólico - foi utilizado.

Durante as análises notamos que as relações foram estabelecidas utilizando-se do RF e/ou do RS, exceto P1 que visualizou somente aspectos figurais e P3, P4 e P12 que não identificaram relações de qualquer tipo entre as etapas do fractal.

A análise apenas do RS, seja na forma numérica ou algébrica, para estabelecer relações entre as etapas do fractal, foi realizada pelo maior número de professores, P2, P5, P6, P7, P8, P9, P10, P11, P13 e P14. Na resposta mencionada por P11, por exemplo, tivemos: "Se observamos o hexágono inicial vemos que 6 lados geram 6 novos polígonos e assim sucessivamente. Como podemos escrever uma potência de base 6". P2, afirma que "Sim, a figura diminui proporcionalmente". Já P8 relata: "O fracionamento das figuras, fazendo relações de razão e proporção". Pelo fato destas relações solicitarem que o registro numérico seja realizado, consideramos que estes professores analisaram apenas este registro.

Apenas P1 encontrou uma relação entre as etapas do fractal, analisando tanto o registro figural quanto o registro simbólico. A seguir, a resposta apresentada por P1: "Sim. Várias: as figuras são simétricas em vários eixos e em diferentes tipos reflexão, rotação e translação; vão diminuindo mantendo suas proporções em relação às medidas aos perímetros; potências em relação às áreas e outras".

Diante destas descrições, concluímos que caso os professores não utilizem diferentes representações semióticas para estabelecer relações entre as medidas de área e perímetro em cada hexágono que surgem nas etapas do fractal, pode ocorrer que estes não solicitem em suas atividades uma exploração que permeie por diferentes representações, como foi o caso dos professores P2 e P10, que requisitaram o registro simbólico na questão 2 e analisaram apenas este registro para estabelecer as relações na questão 3.

Houve também casos, como o dos professores P5 e P12, que estabeleceram relações utilizando o registro numérico, mas não solicitaram conversões em suas atividades elaboradas na questão 2. Já no caso do professor P4 aconteceu o inverso, ou seja, este demandou conversões em sua atividade elaborada para a questão 2, porém não estabeleceu relações utilizando a representação numérica.

Os professores P1, P6, P7, P8, P9, P11, P13 e P14 foram os que estabeleceram relações utilizando o registro simbólico na forma numérica, mas elaboraram atividades para explorar os conteúdos matemáticos que solicitavam a presença do RLN ou do RSN.

\section{Considerações Finais}

O trabalho com a Geometria Fractal atrelada a outros conteúdos matemáticos é algo possível de ocorrer, e esse fato é reconhecido pelos professores, visto que em nossas análises identificamos que os professores participantes acreditam que conteúdos estruturantes, como Funções, Geometria, Grandezas e Medidas, e Números e Álgebra podem ser trabalhados durante e após a construção do Fractal Hexagonal Tipo Dürer. Assim, consideramos a exploração com fractais algo enriquecedor para o processo de ensino e aprendizagem, abrangendo diversas áreas e conceitos da matemática.

Durante a análise da segunda questão do questionário da pesquisa, identificamos que a representação semiótica mais solicitada na atividade elaborada pelos professores foi a numérica e, consequentemente, a maior possibilidade de conversão ocorreu da representação figural do Hexágono de Dürer para a representação numérica. Identificamos, 
também, que houve certa recorrência à língua natural, enquanto a representação algébrica foi solicitada em apenas uma atividade, pelo professor P1.

As DCE destacam a respeito do ensino da álgebra na Educação Básica enfatizando a necessidade de que os alunos a compreendam e possam analisá-la e descrevê-la em diferentes contextos, explorando os significados produzidos a partir destes conteúdos (PARANÁ, 2008).

Diante deste contexto, indicamos que uma das formas de explorar o RF do fractal Hexagonal Tipo Dürer é explorar a generalização algébrica das principais características desse fractal, como por exemplo, do seu perímetro e área. No entanto, destacamos o fato dessa representação ter sido solicitada por somente um professor. Tal incidência pode ser um indicativo para a pouca recorrência a generalizações matemáticas em atividades de geometria nas aulas de Matemática.

Durante a análise da questão 2, observamos também tarefas matemáticas elaboradas com enunciados confusos, sem relação com a matemática, e outros um tanto previsíveis e mecânicos. Isso nos leva a refletir que em alguns momentos o uso do livro didático torna o profissional um tanto dependente e pouco o prepara para elaborar e propor novas ideias de tarefas para serem trabalhadas em sala de aula.

Ressaltamos que abordar a Geometria Fractal em sala de aula e construir seus entes geométricos (fractais), em especial o fractal Hexagonal Tipo Dürer, utilizando um software de Geometria como o GeoGebra, além de proporcionar aulas dinâmicas, proporciona que os professores explorem diversos conteúdos matemáticos, e propicia aos alunos que perpassem por diferentes representações semióticas durante a exploração matemática deste fractal. Ainda, com relação à conversão entre os registros de representação semiótica, concluímos que é possível explorar diferentes conversões entre estes registros ao se trabalhar com uma atividade baseada na construção do Fractal Hexagonal Tipo Dürer.

Com este trabalho, além dos resultados de pesquisa aqui elencados, esperamos ter proporcionado aos professores participantes da pesquisa uma reflexão a respeito da possibilidade de diversidade existente de conteúdos que podem ser trabalhados com uma representação figural na forma de Geometria Fractal, mesmo que isso não tenha sido feito diretamente; e uma exploração do uso do software GeoGebra na construção do fractal Hexagonal tipo Dürer. Afinal, trabalhar a Geometria Fractal, atrelada ao uso do software GeoGebra, e principalmente aos diferentes registros de representação semiótica, proporciona aprendizagens em diferentes campos da Matemática, além de ser atrativo para os alunos, devido ao dinamismo do software e beleza dos fractais.

\section{Referências}

ARAUJO, Elizabeth Adorno de. Ensino de álgebra e formação de professores. Educação Matemática Pesquisa - EMP, São Paulo, v. 10, n. 8, p.331-346, 2008.

BAGIO, Viviane Aparecida. O que dizem as produções paranaenses quanto ao ensino das geometrias não euclidianas a partir da publicação das Diretrizes Curriculares da Educação do Estado do Paraná. Boletim on line de Educação Matemática - BoEM, Joinville, v. 8, n. 4, p.45-65, jul. 2015.

BAGIO, V. A; ROLKOUSKI, E. A implementação das diretrizes curriculares da educação do estado do Paraná e o conteúdo de Geometrias não euclidianas: versões de uma mesma história. In: EPREM 2014, Campo Mourão. Anais do XII Encontro Paranaense de Educação Matemática, 2014. p.1-13. 
BARBOSA, R.M. Descobrindo a geometria Fractal - para a sala de aula. 2. Ed. Belo Horizonte: Autêntica, 2005.

BASNIAK, M. I.; SILVA, S. C. R. Tecnologia em processos culturais de ensino revelados por professores. REnCiMa, v.09, n.04, p.169-182, 2018.

CURY, H.N; LEIVAS, J.C. Atividades com fractais em uma proposta de inovação curricular para cursos de formação de professores. Disponível em: http://limc.ufrj.br/htem4/papers/8.pdf. Acesso em: 22 de outubro de 2018.

CYRINO, M. C.C. T; BALDINI, L. A. F. O software geogebra na formação de professores de matemática - uma visão a partir de dissertações e teses. Revista Paranaense de Educação Matemática RPEM, Campo Mourão, v. 1, n. 1, p.42-61, jun. 2012. Semestral.

DENZIN, N. K.; LINCOLN, Y. S. Introduction: the discipline and practice of qualitative research. In: DENZIN, N. K.; LINCOLN, Y. S. (Eds.). The SAGE handbook of qualitative research. 4. ed. P, 1-19. Thousand Oaks, CA: Sage, 2011.

DUVAL, R. Semiosis y pensamento humano: Registros semióticos y Aprendizajes intelectuales. Trad. Myriam Vega Restrepo. Cali, Colombia: Universidade del Valle, 1999.

. Registros de representação semiótica e funcionamento cognitivo do pensamento. Registres de représentation sémiotique et fonctionnement cognitif de la pensée. Trad. Méricles Thadeu. Revista Eletrônica de Educação Matemática - Revemat [s.I.], v. 7, n. 2, p.266-297, dez. 2012. UFSC.

FARIA. R. W. S; MALTEMPI, M. V. Manipulação e Análise de Padrões Fractais no Processo de Generalização de Conteúdos Matemáticos por meio do Software GeoGebra. In: Conferência Latino Americana de GeoGebra. Anais da Conferência Latino Americana de GeoGebra. PUC, São Paulo, 2012. p.1-15.

FREITAS, J. L. M.; REZENDE, V. Raymond Duval e a Teoria dos Registros de Representação Semiótica Revista Paranaense de Educação Matemática - RPEM. Campo Mourão - PR, Jul-dez, 2013.

MORETTI, M.T. O papel dos registros de representação na aprendizagem matemática.

Contrapontos: Revista do Programa de Pós-Graduação em Educação da Univali. ISSN: 1984-7114. Itajaí, v. 02, n. 6, p. 423-437, 2002.

PARANÁ, Secretaria de Estado da Educação do. Diretrizes Curriculares da Educação Básica de Matemática do Estado do Paraná. Curitiba, 2008.

PIMENTEL, H.; URBAN, P. Fractais da História: a humanidade no caleidoscópio. Editora Masdras Ltda, 1a Edição, 2003.

SILVA, M. J.; ALVES, F. J. C.; NORONHA, C. A. Uma análise semiótica em um ensino de probabilidade com questões sócio críticas. Revista de Ensino de Ciências e Matemática - REnCiMa, v.09, n.04, 2018, p.203-218. 